\title{
Auriculoterapia: evidências científicas sobre a sua eficácia em casos de ansiedade e depressão
}

\author{
Auriculotherapy: scientific evidence about its effectiveness in cases of anxiety and depression \\ Auriculoterapia: evidencia científica sobre su efectividad en casos de ansiedad y depresión \\ Eduardo Viana da Silva ${ }^{1 *}$, Pollyana Sousa Almeida ${ }^{1}$, Deisiane Serrano da Silva ${ }^{1}$, Jéssica Lopes \\ Mota ${ }^{1}$, Calíope Pilger ${ }^{1}$, Ana Carolina Scarpel Moncaio ${ }^{1}$.
}

\section{RESUMO}

Objetivo: Sintetizar o conhecimento científico sobre a eficácia da auriculoterapia em casos de ansiedade e depressão. Métodos: Trata-se de uma Revisão Integrativa de Literatura, buscando responder a seguinte questão norteadora: "quais são as evidências científicas do uso da auriculoterapia no tratamento de ansiedade e depressão". Os critérios de inclusão englobam artigos publicados entre os anos de 2006 a 2021, originais, na íntegra, disponíveis nos idiomas português, inglês e espanhol, e que contemplem a temática central do estudo e, os critérios de exclusão foram publicações secundárias. Utilizaram-se como bases de dados eletrônicas a MEDLINE/Pubmed e LILACS, com os descritores "Auriculoterapia"; "Acupuntura Auricular"; "Ansiedade"; "Depressão" e os correspondentes em inglês. Resultados: A amostra final desta revisão foi composta por seis estudos, que após a sistematização dos estudos selecionados, levantou-se duas categorias temáticas, "A inserção da auriculoterapia como recurso terapêutico nos serviços de saúde" e "A auriculoterapia como uma ferramenta de cuidado integral ao paciente". Os resultados dos estudos levantados demonstraram dados estatísticos significativos nos quadros de depressão e ansiedade em diferentes sujeitos e cenários. Considerações finais: A aplicação da auriculoterapia como intervenção terapêutica em pacientes com depressão e ansiedade, revelou-se eficaz, eficiente e segura.

Palavras-chave: Auriculoterapia, Acupuntura auricular, Ansiedade, Depressão.

\begin{abstract}
Objective: To synthesize scientific knowledge about the effectiveness of auriculotherapy in cases of anxiety and depression. Methods: This is an Integrative Literature Review, seeking to answer the following guiding question: "what is the scientific evidence on the use of auriculotherapy in the treatment of anxiety and depression". Inclusion criteria included articles published between 2006 and 2021, original, in full, available in Portuguese, English and Spanish, and that covered the central theme of the study, and exclusion criteria were secondary publications. MEDLINE/Pubmed and LILACS were used as electronic databases, with the descriptors "Auriculoterapia"; "Acupuntura Auricular"; "Ansiedade"; "Depressão" and the corresponding ones in English. Results: The final sample of this review was composed of six studies, that af ter the systematization of the selected studies, two thematic categories were raised, "The insertion of auriculotherapy as a therapeutic resource in health services" and "Auriculotherapy as a tool for integral patient care". The results of the studies showed significant statistical data on depression and anxiety in different subjects and scenarios. Final considerations: The application of auriculotherapy as a therapeutic intervention in patients with depression and anxiety proved to be effective, efficient, and safe.
\end{abstract}

Key words: Auriculotherapy, Auricular acupuncture, Anxiety, Depression.

1 Universidade Federal de Catalão (UFCAT), Catalão - GO. *E-mail: eduviana@discente.ufcat.edu.br. 


\section{RESUMEN}

Objetivo: Sintetizar los conocimientos científicos sobre la eficacia de la auriculoterapia en casos de ansiedad y depresión. Métodos: Se trata de una revisión bibliográfica integradora, que busca responder a la siguiente pregunta guía: "¿cuál es la evidencia científica sobre el uso de la auriculoterapia en el tratamiento de la ansiedad y la depresión?". Los criterios de inclusión incluyeron artículos publicados entre 2006 y 2021, originales, completos, disponibles en portugués, inglés y español, y que contemplaran el tema central del estudio y los criterios de exclusión fueron publicaciones secundarias. Se utilizaron MEDLINE/Pubmed y LILACS como bases de datos electrónicas, con los descriptores "Auriculoterapia"; "Acupuntura Auricular"; "Ansiedade"; "Depressão" y los correspondientes en inglés. Resultados: La muestra final de esta revisión estuvo compuesta por seis estudios, que tras la sistematización de los estudios seleccionados, se plantearon dos categorías temáticas, "La inserción de la auriculoterapia como recurso terapéutico en los servicios de salud" y "La auriculoterapia como herramienta para la atención integral del paciente". Los resultados de los estudios mostraron datos estadísticos significativos sobre la depresión y la ansiedad en diferentes sujetos y escenarios. Consideraciones finales: La aplicación de la auriculoterapia como intervención terapéutica en pacientes con depresión y ansiedad, demostró ser eficaz, eficiente y segura.

Palabras clave: Auriculoterapia, Acupuntura auricular, Ansiedad, Depresión.

\section{INTRODUÇÃO}

Mundialmente, mais de 450 milhões de pessoas padecem de algum transtorno de saúde mental e os transtornos como a depressão e a ansiedade apresentam-se como um fator relevante de incapacitação na população em nível mundial, restringindo o funcionamento físico, pessoal e social (ORESKOVIC S, 2016; ROCHA ACC, et al., 2021).

A depressão é compreendida por alterações psicopatológicas múltiplas que podem variar conforme a sintomatologia, gravidade e prognóstico. Caracteriza-se pela presença do humor preponderantemente depressivo e/ou irritável e redução da capacidade de sentir satisf ação ou alegria, podendo estar seguidos de uma sensação subjetiva e/ou fadiga, concomitante com mudanças no sono e apetite, desinteresse, pessimismo, lentidão e ideias de fracasso (LIMA AMP, et al., 2016).

Dados da Organização Mundial da Saúde (OMS) (2017) indicam que a quantidade de casos de depressão aumentou 18\% entre 2005 e 2015 acometendo 322 milhões de indivíduos globalmente, sendo a maior parte do sexo feminino. No âmbito nacional, a depressão afeta 11,5 milhões de pessoas, atingindo $5,8 \%$ da população. Concernente à ansiedade, o Transtorno de Ansiedade Generalizada (TAG), caracteriza-se pela preocupação incessante e exagerada, acompanhada de sintomas físicos, como, taquicardia, sudorese, insônia, fadiga, dificuldades de relaxar e dores musculares, sendo que estão associados à hiperatividade autonômica e a tensão muscular, provocando uma repercussão na performance do indivíduo ou um sof rimento considerável (ZUARDI AW, 2017). Ressalta-se que o transtorno de ansiedade abrange múltiplos fatores da personalidade psíquica e emocional do sujeito (D'ÁVILA LI, et al., 2020).

As estatísticas da ansiedade, indicam uma prevalência global de 3,6\%, a qual no continente americano atinge maiores proporções, af etando $5,6 \%$ da população. Destaca-se que no Brasil, este transtorno está presente em 9,3\% da população, compreendendo a maior quantidade de casos entre todos os países do mundo (WORLD HEALTH ORGANIZATION, 2017).

Esses levantamentos epidemiológicos são reverberações da dinâmica da sociedade moderna, que favorecem o desenvolvimento de transtornos mentais e comportamentais, especialmente a ansiedade, 0 estresse e a depressão, que estão sendo frequentes nos atendimentos em saúde (FERNANDES MA, et al., 2018).

Os medicamentos ansiolíticos e antidepressivos são drogas utilizadas para transtornos de ansiedade e depressão, justamente por terem uma ação direta nos neurotransmissores e promover resultados sedativos e tranquilizantes nas atividades do sistema nervoso central. São fármacos tidos como confiáveis, no entanto, 
a utilização desassistida e o uso exacerbado dessas substâncias podem acarretar em riscos à saúde, além de um quadro de dependência (NERI JVD, et al., 2020).

Desta maneira, faz-se necessária outras abordagens terapêuticas objetivando contribuir com o tratamento, com destaque às Práticas Integrativas e Complementares em Saúde (PICS), termo utilizado pelo Ministério da Saúde para a medicina complementar/alternativa, em suas ricas finalidades no Brasil (ANDRADE JT e COSTA LFA, 2010).

As PICS são respaldadas, principalmente, pela Política Nacional de Práticas Integrativas e Complementares no Sistema Único de Saúde (PNPIC-SUS) e pelas portarias n 849/2017 e n 702/2018, que incluíram novas práticas ao rol de terapias integrativas e complementares (MINISTÉRIO DA SAÚDE, 2006; MINISTÉRIO DA SAÚDE, 2017; MINISTÉRIO DA SAÚDE, 2018). Estas constituem um campo de saberes e cuidados e configura-se por um quadro bastante diversificado e sincrético, articulando uma quantidade de métodos diagnósticos-terapêuticos, tecnologias leves, variadas filosofias (como as orientais), práticas religiosas, em recursos sensíveis de experiência corporal e de autoconhecimento. Esses diferentes métodos terapêuticos contemplam artifícios como terapias nutricionais, disciplinas corporais, diversas modalidades de massoterapia, práticas xamânicas e estilos de vida relacionados ao naturalismo e a ecologia (ANDRADE JT e COSTA LFA, 2010).

Dentre esse acervo de estratégias diagnóstico-terapêuticas, evidencia-se a auriculoterapia, conhecida também como acupuntura auricular ou acupressão auricular. Trata-se de uma terapia fundamentada na Medicina Tradicional Chinesa (MTC), utilizada como recurso para diagnosticar e tratar disfunções físicas e psicossomáticas, auxiliando na regulação psíquico-orgânica do paciente, por meio de estímulos em pontos energéticos situados no pavilhão auricular, no qual todo o organismo encontra-se representado como um microssistema. $O$ estímulo dos pontos envolve o reflexo neurológico, neurotransmissores, citocinas, sistema imunológico e inflamação, podendo ser realizados por intermédio de agulhas, esferas de aço, ouro, prata, esferas plásticas ou sementes (HOU PW, et al., 2015).

A auriculoterapia é uma estratégia terapêutica oportuna para tratar casos de ansiedade e depressão e suas fundamentações científicas subsidiam sua eficácia na redução da dor, no tratamento da epilepsia, assim como na obesidade e melhora da qualidade do sono (HOU PW, et al., 2015). Nesse sentido e com base no que foi explicitado, o objetivo deste estudo foi sistematizar o conhecimento científico acerca da eficácia da auriculoterapia em casos de ansiedade e depressão.

\section{MÉTODOS}

Trata-se de uma Revisão Integrativa da Literatura (RIL), estudo que permite a síntese e a investigação de produções científicas, de modo metódico e abrangente, possibilitando a sistematização e propagação do conhecimento produzido (NETO JMR, et al., 2016).

Para o desenvolvimento de uma RIL, é necessário adotar os seguintes passos: 1) elaboração da questão de investigação; 2) busca e seleção dos estudos primários; 3) extração de dados dos estudos; 4) avaliação crítica dos estudos incluídos na revisão; 5) síntese dos resultados da revisão e 6) apresentação da revisão (MENDES KDS, et al., 2019).

A questão norteadora do estudo foi estabelecida de acordo com a estratégia "PICO" (População, Intervenção, Comparação ou Controle e Desfecho ou Resultado). Essa metodologia amplia a probabilidade de que achados científ icos mais adequados para modificar a prática clínica sejam encontrados de forma mais ágil e prática (PAULA CC, et al., 2016). Os componentes da questão de pesquisa, segundo o acrônimo "PICO", foram: População (P) como Indivíduos acometidos por ansiedade e depressão; Intervenção (I) como Auriculoterapia; Comparação (C) como o não uso da auriculoterapia e, Desfecho como eficácia do uso da Auriculoterapia.

Por conseguinte, a questão de pesquisa principal que norteou a revisão integrativa foi a seguinte "quais são as evidências científicas acerca do uso da auriculoterapia no tratamento da ansiedade e depressão?". 
Os critérios de inclusão definidos para seleção foram os seguintes: artigos publicados entre os anos de 2006 a 2021, originais, na íntegra, disponíveis nos idiomas português, inglês e espanhol, e que contemplassem a temática central do estudo. Em contrapartida, os critérios de exclusão adotados foram: publicações secundárias como teses, artigos de opinião, dissertações, manuais e livros.

Definiu-se estudos desde o ano de 2006, levando em consideração que foi o ano de publicação da PNPIC SUS, política que incorporou e implementou o uso de terapias complementares na Atenção Básica (MINISTÉRIO DA SAÚDE, 2006).

Para selecionar os estudos desta RIL, utilizou-se duas bases de dados eletrônicas, sendo elas a Medical Literature Analysis and Retrieval System Online (MEDLINE/PubMed) (via National Library of Medicine) e a Literatura Latino-americana e do Caribe em Ciências da Saúde (LILACS). Elencou-se como palavras-chaves, de acordo com os Descritores em Ciências da Saúde (DeCS): "Auriculoterapia", "Acupuntura Auricular", "Ansiedade", "Depressão", e de acordo com o Medical Subject Headings (MeSH): "Auriculotherapy", Acupuncture, Ear", Anxiety", "Depression". As estratégias de busca abarcaram as combinações entre descritores e os anagramas correspondentes entre os mesmos com o uso do operador booleano "and".

O fluxograma do processo de seleção dos artigos seguiu o Preferred Reporting Items for Systematic Reviews and Meta-Analyses (PRISMA), apresentado a seguir na Figura 1.

Figura 1 - Fluxograma de seleção dos artigos PRISMA
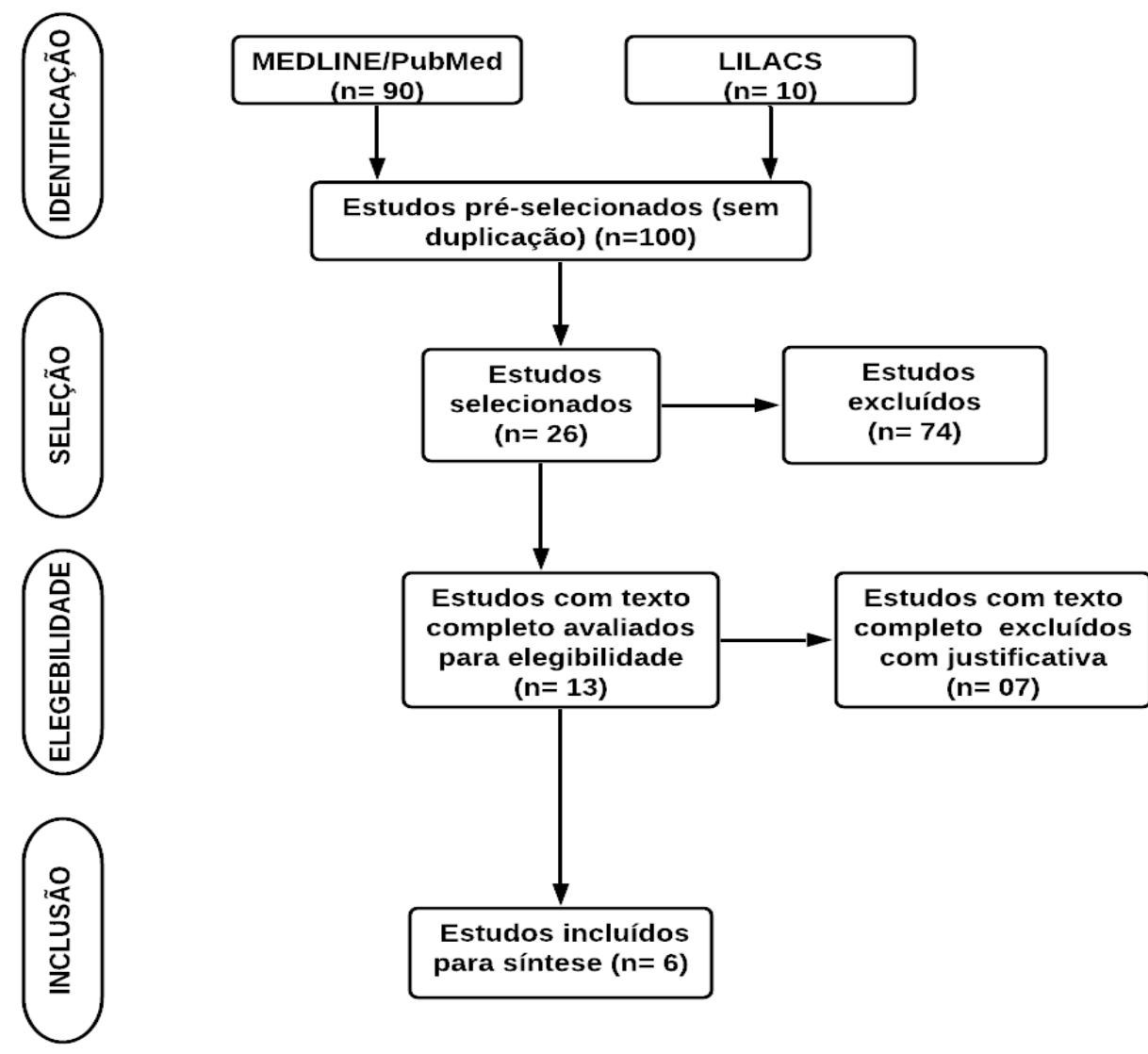

Fonte: Silva EV, et al., 2021.

Os dados levantados devem abranger a amostra do estudo, metodologia proposta, resultados e conclusões do material analisado (MENDES KDS, et al., 2008). Desta maneira, para a extração das informações e estruturação dos dados desenvolveu-se um roteiro contemplando o número do estudo, título, autores, base de dados onde o estudo foi localizado, país, ano, idioma, ano, método utilizado, número da amostra, nível de evidência do estudo, objetivo, resultados, principais conclusões. 
O nível de evidência dos estudos foi avaliado conforme Melnyk BM e Fineout-Overholt E (2011), considerando: o nível I para as revisões sistemáticas ou metanálise, nível II para as evidências derivadas de ensaios clínicos randomizados, nível III para ensaios clínicos sem randomização, nível IV para estudos de coorte e de caso-controle bem delineados, nível V para estudos descritivos e qualitativos, nível VI para evidências derivadas de estudos descritivos ou qualitativos e nível VII para opiniões.

A análise dos dados foi realizada de modo descritivo destacando informações relevantes apontadas nos estudos que compuseram a amostra. Este estudo ocorreu no período de janeiro a agosto de 2021.

\section{RESULTADOS}

A amostra final desta RIL foi composta por seis estudos. Extraíram-se, destes, três (50\%) da base de dados MEDLINE/PubMed e três (50\%) da LILACS. Publicaram-se no que tange ao idioma, quatro (67\%) estudos em inglês e dois (33\%) em português. Identificou-se, no que concerne ao país, três (50\%) publicações no Brasil, duas (33\%) nos Estados Unidos, uma (17\%) na Coreia do Sul. Já em relação ao nível de evidência dos estudos, percebeu-se quatro (66\%) estudos com nível II, um (17\%) estudo com nível III e um (17\%) estudo com nível IV.

O Quadro 1 apresenta as informações extraídas dos estudos conforme roteiro desenvolvido pelos pesquisadores. Identificaram-se os estudos com o número sequencial correspondente precedido pela letra $\mathrm{E}$ (de estudo). 
Quadro 1 - Caracterização dos estudos incluídos, 2021.

\begin{tabular}{|c|c|c|c|c|c|c|c|}
\hline Estudo & Título & Autores (Ano) & $\begin{array}{l}\text { Tipo de estudo/ } \\
\mathrm{n}^{\circ} \mathrm{da} \text { amostra }\end{array}$ & $\begin{array}{l}\text { Nível de } \\
\text { evidência }\end{array}$ & Objetivo & Resultados & Principais conclusões \\
\hline E1 & $\begin{array}{c}\text { Auriculoterapia } \\
\text { em profissionais } \\
\text { de enfermagem } \\
\text { na pandemia do } \\
\text { coronavírus: } \\
\text { estudo de casos } \\
\text { múltiplos }\end{array}$ & $\begin{array}{l}\text { OLIVEIRA CMC, } \\
\text { et al. }(2021)\end{array}$ & $\begin{array}{l}\text { Estudo de caso, } \\
\text { com casos } \\
\text { múltiplos e uma } \\
\text { unidade de } \\
\text { análise/ } 41 \\
\text { profissionais de } \\
\text { saúde }\end{array}$ & Nível IV & $\begin{array}{l}\text { O estudo objetivou analisar o } \\
\text { efeito de uma sessão de } \\
\text { auriculoterapia nos níveis de } \\
\text { ansiedade, depressão e } \\
\text { estresse que afetaram } \\
\text { profissionais de enfermagem } \\
\text { que performaram no decurso } \\
\text { da pandemia da COVID-19 }\end{array}$ & $\begin{array}{l}\text { Observou-se com a sessão de } \\
\text { auriculoterapia que os } \\
\text { profissionais de enfermagem } \\
\text { apresentaram um resultado } \\
\text { considerável sobre as } \\
\text { condições de ansiedade, } \\
\text { depressão e estresse. } \\
\text { Percebeu-se uma redução da } \\
\text { frequência de profissionais } \\
\text { que apresentavam estresse, } \\
\text { ansiedade e depressão no } \\
\text { grau moderado a } \\
\text { extremamente grave, antes da } \\
\text { intervenção, para os níveis } \\
\text { baixos e normal após a } \\
\text { intervenção. No que tange ao } \\
\text { nível de ansiedade, os níveis } \\
\text { mais altos sofreram alteração } \\
\text { para os níveis mais baixos } \\
\text { após a auriculoterapia }\end{array}$ & $\begin{array}{l}\text { Concluiu-se que somente } \\
\text { com uma sessão de } \\
\text { auriculoterapia realizada } \\
\text { com sementes de } \\
\text { mostarda foi possível } \\
\text { alcançar resultados } \\
\text { relevantes no nível de } \\
\text { estresse e na pontuação } \\
\text { das medianas de } \\
\text { ansiedade e depressão. } \\
\text { Portanto, o protocolo } \\
\text { aplicado foi satisfatório } \\
\text { para se obter melhora nos } \\
\text { distúrbios emocionais } \\
\text { pesquisados entre os } \\
\text { profissionais, com base na } \\
\text { significância estatística } \\
\text { entre o antes o o depois } \\
\text { da terapia }\end{array}$ \\
\hline E2 & $\begin{array}{c}\text { Efeitos da } \\
\text { auriculoterapia na } \\
\text { ansiedade de } \\
\text { gestantes no pré- } \\
\text { natal de baixo } \\
\text { risco }\end{array}$ & $\begin{array}{l}\text { SILVA HL, et al. } \\
(2020)\end{array}$ & $\begin{array}{l}\text { Estudo clínico } \\
\text { randomizado, } \\
\text { simples-cego/50 } \\
\text { gestantes }\end{array}$ & Nível II & $\begin{array}{c}\text { O estudo objetivou examinar } \\
\text { os efeitos da auriculoterapia } \\
\text { nos níveis de ansiedade em } \\
\text { gestantes em pré-natal de } \\
\text { baixo risco }\end{array}$ & $\begin{array}{l}\text { Observou-se que a } \\
\text { auriculoterapia é uma terapia } \\
\text { eficaz quando aplicada como } \\
\text { intervenção, tencionando a } \\
\text { redução dos níveis de } \\
\text { ansiedade em gestantes } \\
\text { atendidas em pré-natal de } \\
\text { baixo risco }\end{array}$ & $\begin{array}{l}\text { Concluiu-se que a atuação } \\
\text { do profissional de saúde } \\
\text { acupunturista na } \\
\text { realização de } \\
\text { atendimentos de } \\
\text { gestantes na consulta de } \\
\text { pré-natal de baixo risco é } \\
\text { significativa comparando } \\
\text { os grupos controle } \\
\text { intervenção no que se } \\
\text { refere a redução da } \\
\text { ansiedade }\end{array}$ \\
\hline
\end{tabular}




\begin{tabular}{|c|c|c|c|c|c|c|c|}
\hline Estudo & Título & Autores (Ano) & $\begin{array}{l}\text { Tipo de estudo/ } \\
\text { n }^{\circ} \text { da amostra }\end{array}$ & $\begin{array}{l}\text { Nível de } \\
\text { evidência }\end{array}$ & Objetivo & Resultados & Principais conclusões \\
\hline E3 & $\begin{array}{l}\text { Effectiveness of } \\
\text { auriculotherapy on } \\
\text { anxiety during } \\
\text { labor:a } \\
\text { randomized } \\
\text { clinical trial. }\end{array}$ & $\begin{array}{c}\text { MAFETONIRR, } \\
\text { et al. (2018) }\end{array}$ & $\begin{array}{l}\text { Ensaio clínico } \\
\text { randomizado, } \\
\text { paralelo e triplo- } \\
\text { cego/ } 102 \\
\text { parturientes }\end{array}$ & Nível II & $\begin{array}{l}\text { O estudo objetivou analisar a } \\
\text { efetividade da } \\
\text { auriculoterapia sobre o nível } \\
\text { de ansiedade de parturientes }\end{array}$ & $\begin{array}{c}\text { Observou-se resultados } \\
\text { estatísticos significativos em } \\
\text { todas as análises ao comparar } \\
\text { o grupo de Intervenção versus } \\
\text { Grupo Placebo e Grupo } \\
\text { Intervenção versus Grupo } \\
\text { Controle }\end{array}$ & $\begin{array}{c}\text { Concluiu-se que as } \\
\text { parturientes sujeitadas a } \\
\text { auriculoterapia } \\
\text { mantiveram o controle da } \\
\text { ansiedade no decorrer da } \\
\text { fase ativa do trabalho de } \\
\text { parto, na qual } \\
\text { habitualmente ocorre um } \\
\text { aumento da ansiedade, } \\
\text { como foi percebido nos } \\
\text { Grupo Placebo e Grupo } \\
\text { Controle. A questão de } \\
\text { não ter tido aumento nos } \\
\text { escores de ansiedade no } \\
\text { Grupo de Intervenção, } \\
\text { sugeriu um efeito benéfico } \\
\text { dessa terapia }\end{array}$ \\
\hline E4 & $\begin{array}{c}\text { Auricular } \\
\text { Acupuncture } \\
\text { Versus } \\
\text { Progressive } \\
\text { Muscle Relaxation } \\
\text { in Patients with } \\
\text { Anxiety Disorders } \\
\text { or Major } \\
\text { Depressive } \\
\text { Disorder: } A \\
\text { Prospective } \\
\text { Parallel Group } \\
\text { Clinical Trial }\end{array}$ & $\begin{array}{l}\text { LORENT L, et } \\
\text { al. (2016) }\end{array}$ & $\begin{array}{l}\text { Ensaio clínico } \\
\text { prospectivo de } \\
\text { grupo paralelo/ } \\
162 \text { participantes }\end{array}$ & Nível III & $\begin{array}{l}\text { O estudo objetivou avaliar a } \\
\text { eficácia da acupuntura } \\
\text { auricular conforme o } \\
\text { protocolo National } \\
\text { Acupuncture Detoxification } \\
\text { Association versus } \\
\text { relaxamento muscular } \\
\text { progressivo em pacientes } \\
\text { com transtornos de } \\
\text { ansiedade ou transtorno } \\
\text { depressivo maior }\end{array}$ & $\begin{array}{l}\text { Observou-se que as duas } \\
\text { terapias demonstram efeitos } \\
\text { consideráveis na diminuição } \\
\text { da ansiedade, tensão e } \\
\text { raiva/agressão, justificando a } \\
\text { prática dessas duas } \\
\text { terapêuticas }\end{array}$ & $\begin{array}{l}\text { Sugere-se que a } \\
\text { acupuntura auricular e o } \\
\text { relaxamento muscular } \\
\text { progressivo podem ser } \\
\text { terapias complementares } \\
\text { úteis e igualmente } \\
\text { eficientes no tratamento } \\
\text { de transtornos de } \\
\text { ansiedade e transtorno } \\
\text { depressivo maior }\end{array}$ \\
\hline
\end{tabular}




\begin{tabular}{|c|c|c|c|c|c|c|c|}
\hline Estudo & Título & Autores (Ano) & $\begin{array}{l}\text { Tipo de estudo/ } \\
n^{\circ} \text { da amostra }\end{array}$ & $\begin{array}{c}\text { Nível de } \\
\text { evidência }\end{array}$ & Objetivo & Resultados & Principais conclusões \\
\hline E5 & $\begin{array}{c}\text { Auricular } \\
\text { Acupuncture for } \\
\text { Exam Anxiety in } \\
\text { Medical Students- } \\
\text { A Randomized } \\
\text { Crossover } \\
\text { Investigation }\end{array}$ & $\begin{array}{c}\text { KLAUSENITZ C, } \\
\text { et al. (2016) }\end{array}$ & $\begin{array}{c}\text { Estudo } \\
\text { prospectivo, } \\
\text { randomizado, } \\
\text { controlado com } \\
\text { placebo, } \\
\text { crossovercego } \\
\text { único/40 alunos }\end{array}$ & Nível II & $\begin{array}{l}\text { O estudo objetivou analisar } \\
\text { se a acupuntura auricular } \\
\text { pode diminuir a ansiedade } \\
\text { do exame em estudantes de } \\
\text { medicina em comparação } \\
\text { com o placebo e sem } \\
\text { condições de intervenção } \\
\text { em uma investigação } \\
\text { cruzada randomizada }\end{array}$ & $\begin{array}{l}\text { Observou-se que tanto a } \\
\text { acupuntura auricular quanto o } \\
\text { placebo foram capazes de } \\
\text { diminuir a ansiedade do } \\
\text { exame em comparação com } \\
\text { nenhuma intervenção em } \\
\text { discentes de medicina, ao } \\
\text { mesmo tem po que a terapia } \\
\text { produziu efeitos mais fortes } \\
\text { comparado ao procedimento } \\
\text { placebo. Percebeu-se que o } \\
\text { procedimento placebo } \\
\text { diminuiu a ansiedade no } \\
\text { exame comparado a nenhuma } \\
\text { intervenção, apesar desse } \\
\text { efeito não ter sido tão } \\
\text { satisfatório quanto a } \\
\text { atenuação da ansiedade pré- } \\
\text { exame através da acupuntura } \\
\text { auricular }\end{array}$ & $\begin{array}{c}\text { Concluiu-se que o } \\
\text { procedimento de } \\
\text { acupuntura auricular } \\
\text { utilizado e a intervenção } \\
\text { placebo demonstraram-se } \\
\text { eficientes na diminuição } \\
\text { dos níveis de ansiedade } \\
\text { no exame entre os } \\
\text { estudantes de medicina }\end{array}$ \\
\hline E6 & $\begin{array}{l}\text { Out-of-hospital } \\
\text { auricular } \\
\text { acupressure in } \\
\text { elderpatients with } \\
\text { hip fracture: a } \\
\text { randomized } \\
\text { double-blinded } \\
\text { trial }\end{array}$ & $\begin{array}{l}\text { BARKER R. et } \\
\text { al. (2006) }\end{array}$ & $\begin{array}{c}\text { Estudo controlado } \\
\text { e aleatório/38 } \\
\text { pacientes }\end{array}$ & Nível II & $\begin{array}{l}\text { O estudo objetivou avaliar se } \\
\text { a acupressão auricular pode } \\
\text { reduzir o nível de ansiedade } \\
\text { e dor em um grupo de } \\
\text { idosos com fratura aguda de } \\
\text { quadril durante o } \\
\text { atendimento extra-hospitalar } \\
\text { a caminho do hospital }\end{array}$ & $\begin{array}{l}\text { Observou-se que os idosos } \\
\text { que receberam a acupressão } \\
\text { como o verdadeiro grupo de } \\
\text { intervenção tiveram escores } \\
\text { de ansiedade } \\
\text { substancialmente menores na } \\
\text { chegada ao hospital, além de } \\
\text { menor dor e uma frequência } \\
\text { cardíaca mais baixa em } \\
\text { comparação com os idosos } \\
\text { que receberam a intervenção } \\
\text { de controle simulado }\end{array}$ & $\begin{array}{l}\text { Concluiu-se que a } \\
\text { acupressão auricular } \\
\text { aplicada em três pontos } \\
\text { específicos é eficiente na } \\
\text { diminuição da dor e da } \\
\text { ansiedade em pacientes } \\
\text { idosos que sofreram } \\
\text { fratura de quadril }\end{array}$ \\
\hline
\end{tabular}

Fonte: Silva EV, et al., 2021. 
Percebeu-se, com a leitura, análise e extração das informações dos estudos, a necessidade de apresentar elementos específicos em relação à abordagem terapêutica utilizada em cada estudo. O Quadro 2 e Quadro $\mathbf{3}$ apresentam esses dados.

Quadro 2 - Representação dos dados dos artigos referentes ao número de sessões, participantes e escalas utilizadas nos estudos para avaliação de ansiedade e/ou depressão.

\begin{tabular}{|c|c|c|c|}
\hline Estudo & $\begin{array}{c}\mathbf{N}^{\circ} \text { de } \\
\text { Sessões }\end{array}$ & Participantes & Escalas aplicadas \\
\hline E1 & 1 sessão & $\begin{array}{c}\text { Profissionais de enf ermagem } \\
\text { atuantes na pandemia da COVID-19 }\end{array}$ & $\begin{array}{c}\text { Depression, Anxiety, and Stress Scale-21 } \\
\text { (DASS-21) }\end{array}$ \\
\hline E2 & 3 sessões & $\begin{array}{c}\text { Gestantes em pré-natal de baixo } \\
\text { risco }\end{array}$ & $\begin{array}{c}\text { Inventário de Ansiedade Traço-Estado } \\
\text { (IDATE) }\end{array}$ \\
\hline E3 & 1 sessão & Mulheres durante o trabalho de parto & Escala de Ansiedade de Hamilton (HAM-A) \\
\hline E4 & 8 sessões & $\begin{array}{c}\text { Pacientes com diagnóstico primário } \\
\text { de Transtorno de Ansiedade ou } \\
\text { Transtorno Depressivo Maior }\end{array}$ & $\begin{array}{c}\text { Escala Visual Analógica (EVA) de 0-100 mm: } \\
\text { "tensão", "ansiedade", "raiva / agressão" }\end{array}$ \\
\hline E5 & 1 sessão & Estudantes de medicina & $\begin{array}{c}\text { Escala Visual Analógica (EVA) de 0-100 mm: } \\
\text { ansiedade e versão alemã do Inventário de } \\
\text { Ansiedade Traço-Estado de Spielberger } \\
\text { (STAI) (IDATE) }\end{array}$ \\
\hline E6 & 1 sessão & $\begin{array}{c}\text { Pacientes idosos consecutivos que } \\
\text { sofreram fraturas de quadril }\end{array}$ & $\begin{array}{c}\text { Escala Visual Analógica (EVA) de 0-100 mm: } \\
\text { ansiedade }\end{array}$ \\
\hline
\end{tabular}

Fonte: Silva EV, et al., 2021.

Quadro 3 - Abordagem terapêutica utilizada pelos pesquisadores nos estudos.

\begin{tabular}{|c|c|c|c|}
\hline Estudo & $\begin{array}{c}\text { Profissional } \\
\text { Auriculoterapeuta }\end{array}$ & Pontos aplicados & Método de aplicação \\
\hline E1 & $\begin{array}{c}\text { Um estudante e os outros dois } \\
\text { com formação em } \\
\text { enf ermagem, } \\
\text { todos com habilitação em } \\
\text { auriculoterapia } \\
\end{array}$ & $\begin{array}{l}\text { Shen Men, Rim, Sistema Nervoso } \\
\text { Simpático (SNV), Alegria, Ansiedade, } \\
\text { Antidepressivo, Coração, Endócrino, } \\
\text { Pulmão e Relaxamento Muscular }\end{array}$ & Sementes de mostarda \\
\hline E2 & $\begin{array}{c}\text { Enf ermeiro obstetra, } \\
\text { especialista em acupuntura e } \\
\text { eletroacupuntura }\end{array}$ & $\begin{array}{c}\text { Triângulo Cibernético (Shen Men, } \\
\text { Rim e Simpático), Tronco Cerebral, } \\
\text { Baço e Ansiedade }\end{array}$ & $\begin{array}{l}\text { Sementes de mostarda } \\
\text { amarela-colza }\end{array}$ \\
\hline E3 & $\begin{array}{l}\text { Pesquisador principal } \\
\text { responsável que passou por } \\
\text { dois cursos de curta duração }\end{array}$ & $\begin{array}{c}\text { Grupo intervenção: Shen Men, útero, } \\
\text { área de neurastenia, endócrino. } \\
\text { Grupo placebo: tornozelo, joelho, } \\
\text { dente e mandíbula }\end{array}$ & $\begin{array}{l}\text { Grupo de intervenção: } \\
\text { microesferas de cristais } \\
\text { polidos. Grupo placebo: } \\
\text { microesferas de vidro, } \\
\text { semelhante as de cristais }\end{array}$ \\
\hline E4 & $\begin{array}{c}\text { Acupunturistas enfermeiras, } \\
\text { especialmente treinadas e } \\
\text { certificadas na teoria e prática } \\
\text { do uso do protocolo National } \\
\text { Acupuncture Detoxification } \\
\text { Association }\end{array}$ & $\begin{array}{l}\text { Simpático, Shen Men, rim, fígado e } \\
\text { pulmão }\end{array}$ & Agulhamento \\
\hline E5 & $\begin{array}{c}\text { Acupunturista licenciado com } \\
\text { mais de cinco anos de } \\
\text { experiência }\end{array}$ & $\begin{array}{c}\text { Grupo intervenção: Pulmão, Shen } \\
\text { Men, rim, subcórtex, glândula } \\
\text { adrenal. Grupo Placebo: cinco locais } \\
\text { na hélice da orelha }\end{array}$ & $\begin{array}{l}\text { Grupo intervenção e } \\
\text { placebo: agulhamento } \\
\text { bilateral }\end{array}$ \\
\hline E6 & $\begin{array}{c}\text { Paramédico sem } \\
\text { conhecimento ou experiência } \\
\text { com acupressão ou } \\
\text { tratamentos semelhantes, no } \\
\text { entanto foi intensamente } \\
\text { treinado por um médico com } \\
\text { experiência em acupressão e } \\
\text { acupuntura } \\
\end{array}$ & $\begin{array}{l}\text { Grupo intervenção verdadeira: Shen } \\
\text { Men, quadril, ponto de Valium. Grupo } \\
\text { de controle simulado: ponta da } \\
\text { concha }\end{array}$ & $\begin{array}{l}\text { Grupo de intervenção } \\
\text { verdadeira e Grupo de } \\
\text { controle simulado: } \\
\text { esferas de plástico para } \\
\text { acupressão (bilateral) }\end{array}$ \\
\hline
\end{tabular}

Fonte: Silva EV, et al., 2021. 
Vislumbrou-se, na sistematização dos estudos selecionados, a construção de duas categorias temáticas: "A inserção da auriculoterapia como recurso terapêutico nos serviços de saúde" e "A auriculoterapia como uma ferramenta de cuidado integral ao paciente".

\section{DISCUSSÃO}

Incluíram-se, na categoria "A inserção da auriculoterapia como recurso terapêutico nos serviços de saúde", quatro estudos (67\%), sendo eles E1, E2, E3 e E6. Estes estudos se relacionam por possuírem como campo de pesquisa serviços de saúde, no entanto, serviços inseridos em diferentes níveis de atenção em saúde (OLIVEIRA CMC, et al., 2021; SILVA HL, et al., 2020; MAFETONIR R, et al., 2018; BARKER R, et al., 2006).

No que tange ao estudo E1, a amostra do estudo foi composta por profissionais de enfermagem de um hospital universitário que estavam atuando na linha de frente da pandemia da COVID-19, os pesquisadores identificaram, após a $1^{\circ}$ sessão de auriculoterapia com o uso de sementes, um ef eito benéfico significativo nas variáveis ansiedade, depressão e estresse. Percebeu-se uma significância estatística entre o antes e depois da intervenção auricular, havendo reduções expressivas do nível de estresse e na pontuação das medianas de ansiedade e depressão (OLIVEIRA CMC, et al., 2021).

Cabe salientar, que no cenário pandêmico verificou-se uma alta prevalência de sintomas severos de ansiedade e depressão entre os profissionais de enfermagem que atuam em serviços de média e alta complexidade (SANTOS KMR, et al., 2021). Além disso, os profissionais de enfermagem constituem um dos grupos mais acometidos devido a pandemia, considerando o risco de contágio e a dor emocional que compromete significativamente a saúde mental (HUMEREZ DC, et al., 2020).

Concernente aos estudos E2 e E3, ambos avaliaram o ef eito da auriculoterapia no âmbito da obstetrícia. No estudo E2, compuseram a amostra do estudo gestantes atendidas em pré-natal de baixo risco de uma maternidade filantrópica. Os pesquisadores perceberam com três sessões utilizando sementes a eficácia da auriculoterapia como artifício terapêutico para redução dos níveis de ansiedade e também encontrou-se um resultado significativo na comparação entre o grupo controle e intervenção no que concerne a redução da ansiedade (SILVA HL, et al., 2020; MAFETONIR R, et al., 2018).

Relativo ao estudo E3, a intervenção foi realizada com mulheres em trabalho de parto em um hospital universitário com o uso de microesferas de cristais polidos. Os pesquisadores identificaram após a $1^{\circ}$ sessão que a técnica se apresentou eficaz na diminuição dos sintomas de ansiedade, além do mais, os resultados demonstraram-se estatisticamente significativos ao comparar o grupo de intervenção ao grupo placebo. Outrossim, perceberam um aumento no nível de ansiedade nos grupos placebo e grupo controle, enquanto no grupo intervenção que recebeu a terapia adequada não ocorreu esse aumento, revelando um ef eito válido da auriculoterapia (MAFETONIR R, et al., 2018).

Ressalta-se que as PICS vem sendo empregadas na fase ativa do trabalho de parto, dentre elas a auriculoterapia, que demonstra ter benefícios na diminuição da dor, ansiedade e redução do tempo de trabalho de parto (SILVA ADV, et al., 2020).

No que se refere ao estudo E6, a pesquisa foi realizada no âmbito do atendimento pré-hospitalar em serviços de urgência e emergência, no qual a intervenção auricular foi realizada durante o transporte de ambulância com pacientes que sofreram fratura do quadril. Os pesquisadores perceberam após a $1^{\circ}$ sessão com esferas de plástico para acupressão que o grupo intervenção teve escores de ansiedade consideravelmente mais baixos na chegada ao hospital, em comparação com os pacientes do grupo controle. Desse modo, a auriculoterapia demonstrou-se eficaz na redução da dor e da ansiedade (BARKER R, et al., 2006).

Observou-se que os estudos obtiveram resultados e conclusões análogas sobre a eficácia da auriculoterapia em casos de depressão e principalmente de ansiedade. A utilização da intervenção auricular foi percebida em serviços, desde a Atenção Primária à Saúde (APS) até serviços de atenção terciária ou de alta complexidade, evidenciando a versatilidade desta abordagem terapêutica (OLIVEIRA CMC, et al., 2021; SILVA HL, et al., 2020; MAFETONIR R, et al., 2018; BARKER R, et al., 2006). 
Considerando o cenário pandêmico atual e as diversas repercussões para o estilo de vida da população, alguns fatores como o isolamento social e, consequentemente, o tempo de estadia das crianças em domicílio, o desenvolvimento do trabalho home office, a necessidade da utilização de máscaras foram condições importantes para o aumento dos sintomas de ansiedade. Além disso, uma outra variável que favoreceu a potencialização do quadro de ansiedade, compete-se ao medo e a insegurança das pessoas frente a grandeza de informações sobre a COVID-19 (ROLIM JA, et al., 2020).

Os números de diagnóstico de ansiedade, que constantemente se manifestam com disfunções do sono e comorbidade com depressão, aumentaram no curso da pandemia da COVID-19 (NICOLINI H, 2020). PérezCano HJ, et al. (2020) identificaram que os indicadores emocionais, como ansiedade, depressão e estresse provenientes da pandemia do novo coronavírus foram apresentados em quase metade da amostra em seu estudo.

Cabe destacar, que nesse cenário existe uma alta prevalência da utilização indiscriminada de ansiolíticos no âmbito nacional, justificado por diversos fatores, como a falta de conhecimento do diagnóstico, da terapêutica, do tempo de ação do fármaco e dos eventos adversos. Os principais medicamentos utilizados são os benzodiazepínicos e os antidepressivos. Convém destacar, que essas drogas estão disponibilizadas em todas as farmácias e que a prescrição sem análise clínica completa do paciente pode acarretar no uso indiscriminado destes medicamentos e causar dependência, a qual pode acarretar em agravos na qualidade de vida dos pacientes (FELIX FJ, et al., 2021).

Nesta perspectiva, faz-se necessário uma terapia complementar eficaz e não farmacológica que contribua no tratamento de casos de ansiedade e depressão. Em um estudo realizado por Landgren K, et al. (2019) objetivou-se descrever as percepções dos profissionais de saúde diante da aplicação da auriculoterapia como recurso terapêutico complementar na atenção psiquiátrica. Esses profissionais que oferecem a intervenção auricular como parte do cuidado descrevem a técnica como um complemento simples, seguro, prático e promissor no alívio de sintomas psiquiátricos e somáticos. Ademais, apontam que a terapia propicia uma calma interior e resulta em um ef eito positivo em diversos sintomas de seus pacientes.

A inserção da auriculoterapia nos serviços de saúde contempla todos os níveis de atenção, revelando-se como uma técnica que pode ser utilizada desde à atenção básica até em clínicas e hospitais de alta complexidade. Além disso, a terapia auricular como uma das PICS tem como fundamento uma perspectiva integradora, considerando o ser humano nas dimensões física, mental, emocional e espiritual. Desse modo, incluíram-se, na categoria "A auriculoterapia como uma ferramenta de cuidado integral ao paciente", dois estudos (33\%), sendo eles E4 e E5, onde são abordados tais aspectos (LORENT L, et al., 2016; KLAUSENITZ C, et al., 2016).

No que tange ao estudo $\mathrm{E} 4$, a amostra foi composta por pacientes de uma enf ermaria psiquiátrica com diagnóstico primário de transtorno de ansiedade ou transtorno depressivo maior. Nesta pesquisa, o objetivo foi avaliar a eficácia da auriculoterapia aplicada conforme o protocolo padronizado National Acupuncture Detoxification Association (NADA) versus a terapia de relaxamento muscular progressivo. Os pesquisadores perceberam após oito sessões com o uso de agulhas, que a intervenção auricular demonstrou ef eitos significativos na ansiedade, tensão e raiva/agressão no decorrer de todas as semanas. Destaca-se que houve uma melhora significativa após a terapia em cada semana. Outro estudo realizado com profissionais do sistema penitenciário percebeu-se com três sessões resultados suficientes para reduzir em $33,3 \%$ os sintomas de estresse em grau extremo, além de reduzir a intensidade da ansiedade após três e seis sessões (GRAÇA BC, et al., 2020).

Concernente ao estudo E5, a intervenção realizada por técnica de agulhamento foi feita em estudantes de medicina de uma universidade de Greifswald que seriam submetidos a três exames de anatomia oral em um mês. Os pesquisadores identificaram, após a $1^{\circ}$ sessão, uma redução do nível de ansiedade no grupo intervenção em comparação com o grupo placebo e grupo sem intervenção. Além disso, no grupo placebo também houve uma redução da ansiedade se comparado ao grupo nenhuma intervenção, apesar de que esse ef eito não foi tão significativo quanto a diminuição da ansiedade pré-exame por meio da auriculoterapia. Destaca-se que os estudantes de medicina são um público onde a prevalência dos sintomas de ansiedade e 
depressão torna-se constante e sua incidência aumenta consideravelmente. De maneira ampla, sabe-se que as taxas de transtornos psíquicos nesse público são maiores do que as da população em geral e de outros grupos universitários (MACHADO SLM, et al., 2019).

Avaliou-se nos estudos E4 e E5, os aspectos relacionados ao contexto emocional, como ansiedade, tensão, raiva/agressão, buscando verificar a eficácia da auriculoterapia diante de tais fatores. Ambos os estudos corroboram e reforçam a ef etividade da técnica, portanto, percebe-se que a auriculoterapia promove ef eitos que extrapolam a dimensão física do paciente, interferindo e colaborando de modo resolutivo em questões mentais e emocionais (LORENT L, et al., 2016; KLAUSENITZ C, et al., 2016).

As PICS, dentre as quais inclui-se a auriculoterapia, são terapias que apresentam uma alta demanda por parte dos usuários, havendo uma explícita compreensão dos seus benefícios. Evidências científicas apontam repercussões positivas na saúde dos usuários nas dimensões psicológica, física e emocional, além de apresentar os benefícios da utilização particular dessas práticas por indivíduos com patologias crônicas (DACAL MPO e SILVA IS, 2018).

Nesse sentido, vale reforçar as dificuldades no tratamento de ansiedade e depressão. Cruz LP, et al. (2016) apontaram empecilhos para a manutenção da terapia medicamentosa entre pessoas com transtorno de ansiedade, como: desconhecimento sobre o diagnóstico e tratamento, preocupações, temores e insatisfação com os ef eitos dos medicamentos, falhas no fornecimento dos fármacos, inaptidão para administração do próprio medicamento e a necessidade de um atendimento que não se reduza à abordagem medicamentosa. Além disso, com base no estudo, os autores afirmaram que um atendimento reducionista e com propósitos restritos a farmacoterapia podem dificultar a adesão aos medicamentos.

Ao mesmo tempo, lbanez $G$, et al. (2014) identificaram as dificuldades no tratamento medicamentoso em pacientes com depressão, percebendo aspectos como a repercussão do s fenômenos depressivos sobre a aptidão para cuidar de si, o descontentamento com as implicações da terapia medicamentosa, a fragilidade do paciente e carecimento de suporte, o uso de diversos medicamentos concomitantemente e a falta de informações sobre a patologia e o tratamento.

Em ambas as doenças, tanto em pacientes com ansiedade, quanto com depressão, pode-se evidenciar questões emocionais, sociais e comportamentais que acabam impactando negativamente no tratamento. Assim, reduzir o tratamento somente à intervenção farmacológica pode vir a interferir prejudicialmente na melhora do paciente (CRUZ LP, et al., 2016; IBANEZ G, et al., 2014).

Desse modo, a auriculoterapia e as PICS, surgem como terapias que podem ser acionadas, buscando uma assistência em saúde integral e humanizada, complementando o tratamento convencional. Assim, potencializam os resultados do tratamento e promovem uma melhora mais efetiva no quadro de saúde do paciente. A auriculoterapia amplia os dispositivos de cuidado recorrente ao paciente, de forma acessível e natural, estimulando as forças curativas do organismo e gerando resultados cong ruentes, sem desconsiderar a medicina alopática, que segmenta o cuidado e objetiva a cura física. É importante considerar o ser humano multidimensional, integrando o físico, a mente, o espírito e o emocional (FREITAG VL e KRAUSE KMO, 2019).

Convém salientar que a frequência de aplicação pode interf erir na resolutividade terapêutica. A assistência com as PICS permitem a diminuição evidente da intensidade dos sintomas com um número reduzido de sessões (cinco), ao mesmo tempo que é possível perceber que a extensão da assistência com a terapia auricular aspira proporcionar maiores resultados, visto que a intensidade da melhora se manifesta com 0 avanço do acompanhamento terapêutico (DACAL MPO e SILVA IS, 2018).

\section{CONSIDERAÇÕES FINAIS}

A aplicação da auriculoterapia como intervenção terapêutica em pacientes com depressão e ansiedade, revelou-se eficaz, eficiente e segura. Os dados dos estudos demonstram resultados estatísticos significativos nos quadros de depressão e ansiedade em diferentes sujeitos e cenários. Com base nos níveis de evidência dos estudos que compuseram a amostra, existe uma prevalência de ensaios clínicos randomizados bem delineados, demonstrando um rigor metodológico que constata a fidedignidade dos resultados. Deste modo, 
esta revisão visou contribuir com a terapêutica de casos de ansiedade e depressão, além de colaborar para o crescimento e fortalecimento das PICS.

\section{REFERÊNCIAS}

1. ANDRADE JT, COSTA LFA. Medicina Complementar no SUS: práticas integrativas sob a luz da Antropologia médica. Saúde Soc., 2010;19(3): 497-508.

2. BARKER R, et al. Out-of-hospital auricular acupressure in elder patients with hip fracture: a randomized doubleblinded trial. Acad. Emerg. Med., 2006;13(1): 19-23.

3. MINISTÉRIO DA SAÚDE. Portaria $n^{\circ} 702$, de 21 de março de 2018. 2018. Disponível em: https://bvsms.saude.gov.br/bvs/saudelegis/gm/2018/prt0702_22_03_2018.html. Acesso em :24 jul. 2021.

4. MINISTÉRIO DA SAÚDE. Portaria $n^{\circ} 849$, de $2 \overline{7}$ de março de 2017.2017. Disponível em: https://bvsms.saude.gov.br/bvs/saudelegis/gm/2017/prt0849_28_03_2017.html. Acessado em:24 de julho de 2021.

5. MINISTÉRIO DA SAÚDE. Secretaria de Atenção à Saúde. Departamento de Atenção Básica. Política Nacional de Práticas Integrativas e Complementares no SUS (PNPIC-SUS). 2006. Disponível em: http://189.28.128.100/dab/docs/publicacoes/geral/pnpic.pdf. Acessado em:24 de março de 2021.

6. CRUZ LP, et al. Dificuldades relacionadas à terapêutica medicamentosa no transtorno de ansiedade. Rev. Eletr. Enf., 2016;18: e1155.

7. D'ÁVILA LI, et al. Processo Patológico do Transtorno de Ansiedade Segundo a Literatura Digital Disponível em Português - Revisão Integrativa. Revista Psicologia e Saúde, 2020; 12(2):155-168.

8. DACAL MPO, SILVA IS. Impactos das práticas integrativas e complementares na saúde de pacientes crônicos SAÚDE DEBATE, 2018; 42 (118): 724-735.

9. FELIX FJ, et al. Ansiedade e o uso indiscriminado de ansiolíticos. REBES, 2021;11(1): 49-55.

10. FERNANDES MA, et al. Prevalência dos transtornos de ansiedade como causa de afastamento de trabalhadores. Rev Bras Enferm., 2018;71(Suppl.5): 2344-51.

11. FREITAG VL, KRAUSE KMO. Trajetória da implantação de auriculoterapia na rede municipal de saúde de Cruz Alta - Rio Grande do Sul. Revista Espaço Ciência e Saúde, 2019;7(2): 42-51.

12. GRAÇA BC, et al. Uso da auriculoterapia no controle da lombalgia, ansiedade estresse de profissionais do sistema penitenciário. BrJP, 2020;3(2): 42-6.

13. HOU PW, etal. The History, Mechanism, and Clinical Application of Auricular Therapy in Traditional Chinese Medicine. Evidence-based Complementary and Alternative Medicine, 2015;1-13.

14. HUMEREZ DC, et al. Saúde mental dos profissionais de enfermagem do Brasil no contexto da pandemia COVID-19: ação do conselho federal de enfermagem. Cogitare enferm., 2020;25: e74115.

15. IBANEZ G, et al. Adesão e dificuldades relacionadas ao tratamento medicamentoso em pacientes com depressão. Rev Bras Enferm., 2014;67(4):556-62.

16. KLAUSENITZ C, et al. Auricular Acupuncture for Exam Anxiety in Medical Students-A Randomized Crossover Investigation. PLoS One, 2016; 11(12):1-9.

17. LANDGREN K, et al. Ear Acupuncture in Psychiatric Care From the Health Care Professionals' Perspective: A Phenomenographic Analysis. Issues Ment Health Nurs., 2019; 40(2): 166-175.

18. LIMA AMP, et al. Depressão em idosos: uma revisão sistemática da literatura. R Epidemiol Control Infec, 2016;6(2): 97-103.

19. LORENT L, et al. Auricular Acupuncture Versus Progressive Muscle Relaxation in Patients with Anxiety Disorders or Major Depressive Disorder: A Prospective Parallel Group Clinical Trial. J. Acupunct Meridian Stud., 2016; 9(4): 1919.

20. MACHADO SLM, et al. Ansiedade e depressão em estudantes de medicina. Revista Saúde Multidisciplinar, 2019; 2(6): $1-5$.

21. MAFETONI RR, et al. Effectiveness of auriculotherapy on anxiety during labor: a randomized clinical trial. Rev. LatinoAm. Enfermagem, 2018;26:e3030.

22. MELNYK BM, FINEOUT-OVERHOLT E. Evidence-based practice in nursing \& healthcar: a guide to best practice. 2nd ed. Filadélfia:Lippincott Williams \& wilkins, 2011;599p.

23. MENDES KDS, et al. Revisão Integrativa: método de pesquisa para a incorporação de evidências na saúde e na enfermagem. Texto \& contexto enferm., 2008;17(4): 758-64.

24. MENDES KDS, et al. Uso de gerenciador de referências bibliográficas na seleção dos estudos primários em revisão integrativa. Texto \& contexto enferm.,2019;28: e20170204.

25. MOHER D, et al. Preferred Reporting Items for Systematic Reviews and Meta-Analyses: The PRISMA Statement. PLoS Med, 2009;6(7): 1-6.

26. NERI JVD, et al. M. Uso de ansiolíticos e antidepressivos por acadêmicos da área da saúde: uma revisão bibliográfica. Braz. J. of Develop., 2020; 6(10): 75673-75686

27. NETO JMR, et al. Análise de teorias de enfermagem de Meleis: revisão integrativa. Rev. Bras. Enferm.,2016;69(1): 174-81.

28. NICOLINI H. Depresión y ansiedad en los tiempos de la pandemia de COVID-19. Cir Cir., 2020;88(5): $542-547$.

29. OLIVEIRA CMC, et al. Auriculoterapia em profissionais de enfermagem na pandemia do coronavírus: estudo de casos múltiplos. Rev. Eletr. Enferm., 2021;23(65678):1-9. 
30. ORESKOVIC S. Breaking down the Silo Mentality in Global Mental Health: The New Role for the Schools of Public Health. Psychiatr Danub., 2016; 28(4): 318-20.

31. PAULA CC, et al. Revisão integrativa como ferramenta para tomada de decisão na prática de saúde. In: LACERDA MR, COSTENARO RGS. Metodologias da pesquisa para a enfermagem e saúde: da teoria à prática. Porto Alegre: Moriá; 2016;496p.

32. PÉREZ-CANO HJ, et al. Anxiety, depression, and stress in response to the coronavirus disease-19 pandemic. Cir Cir., 2020; 88(5):562-568.

33. ROCHA ACC, et al. Impacto dos transtornos depressivo e de ansiedade no controle da asma. Rev Med., 2021; 100(3): 128-34.

34. ROLIM JA, et al. Manejo da Ansiedade no Enfrentamento da Covid-19. Rev Enfermagem e Saúde Coletiva, 2020; 4(2): 64-74.

35. SANTOS KMR, et al. Depressão e ansiedade em profissionais de enfermagem durante a pandemia da COVID -19. Escola Anna Nery, 2021;25: e20200370.

36. SILVA ADV, et al. Os benefícios das práticas integrativas e complementares no trabalho de parto. Research, Society and Development, 2020;9(7):1-16.

37. SILVA HL, et al. Efeitos da auriculoterapia na ansiedade de gestantes no pré-natal de baixo risco. Acta Paul Enferm., 2020;33: eAPE20190016.

38. WORLD HEALTH ORGANIZATION. Depression and other common mental disorders: global health estimates. 2017. Disponível http://apps.who.int/iris/bitstream/handle/10665/254610/;jessionid=E0E42C227F9ABE18C5A8EBCE84D198D9?seq uence $=1$. Acessado em: 24 de julho de 2021 .

39. ZUARDI AW. Características básicas do transtorno de ansiedade generalizada. Medicina, 2017;50(Supl. 1): 51-55. 